\title{
The T Tauri star RX J1608.6-3922 - not an eclipsing binary but a spotted single star ${ }^{\star}$
}

\author{
V. Joergens ${ }^{1}$, E. Guenther ${ }^{2}$, R. Neuhäuser ${ }^{1}$, M. Fernández ${ }^{3}$, and J. Vijapurkar ${ }^{4}$ \\ 1 Max-Planck-Institut für Extraterrestrische Physik, Giessenbachstrasse 1, 85748 Garching, Germany \\ 2 Thüringer Landessternwarte Tautenburg, Karl-Schwarzschild-Observatorium, Sternwarte 5, 07778 Tautenburg, \\ Germany \\ 3 Instituto de Astrofísica de Andalucía (CSIC), Apdo. 3004, 18080 Granada, Spain \\ 4 Homi Bhabha Centre for Science Education (Tata Inst. of Fundamental Research), V. N. Purav Marg Mankhurd, \\ Mumbai 400088, India
}

Received 13 March 2001 / Accepted 10 May 2001

\begin{abstract}
High-resolution spectroscopy and photometric monitoring of the pre-main sequence star RX J1608.63922 shows that it is not an eclipsing binary, as previously claimed. Radial velocity measurements covering suitable time spans in order to detect a spectroscopic binary with the claimed period of about 7 days have been performed. The scatter of the radial velocity does not exceed $2.4 \mathrm{~km} \mathrm{~s}^{-1}$, defining an upper mass limit of $24 M_{\text {Jupiter }}$ for any eclipsing companion orbiting this star with the claimed period. Photometric observations of RX J1608.6-3922 in 7 consecutive nights (i.e. as long as the claimed orbital period) reveal brightness variations of the order of $0.2 \mathrm{mag}$ with a period of 3.6 days. The shape of the detected light curve differs from a light curve of the star recorded in 1996. The small variations of the radial velocity, the variable shape of the light curve, as well as $(B-V)$ color variations suggest that the flux of RX J1608.6-3922 is modulated by spots on the stellar surface with a rotational period of 3.6 days. The stellar activity of this star seems to be highly variable, taking into account the variable shape of the light curve, with an amplitude varying from 0.5 to $0.2 \mathrm{mag}$ in a few years, as well as hints for a variable $\mathrm{H}_{\alpha}$ equivalent width.
\end{abstract}

Key words. binaries: eclipsing - binaries: spectroscopic - stars: individual: RX J1608.6-3922 stars: pre-main-sequence - stars: rotation - starspots

\section{Introduction}

Photometric observations of the young, pre-main-sequence (PMS) object RX J1608.6-3922 revealed brightness variations typical for an eclipsing system (Wichmann et al. 1998, hereafter W98). The light curve recorded in 1996 in the $V$ band in nine consecutive nights displays two deep minima of the order of $0.5 \mathrm{mag}$ and $0.3 \mathrm{mag}$ and the authors found a period of 7.2 days. Similar amplitudes of a primary and a secondary eclipse indicate similar luminosities and therefore a mass ratio near unity. Such systems are very likely double-lined spectroscopic binaries (SB2).

The detection of an eclipsing SB2 pre-main-sequence star would be highly interesting since it would allow the direct determination of the masses of the two stellar components. The mass is the most fundamental parameter for

\footnotetext{
Send offprint requests to: V. Joergens,

e-mail: viki@mpe.mpg.de

* Based on observations obtained at the European Southern Observatory at La Silla, Chile in program 62.I-0418, 63.I0096, 64.I-0294, 65.I-0275 and on observations obtained at the MSSSO Observatory at Siding Springs, Australia.
}

the evolution of a star and is therefore a very important input parameter for evolutionary theories. So far there are only a few late-type low-mass PMS systems known with accurately determined masses: an eclipsing SB2 in Orion, RX J0529.4+0041, with stellar masses of $1.30 M_{\odot}$ and $0.95 M_{\odot}$ (Covino et al. 2000); a $1.6 M_{\odot}$ T Tauri star in an eclipsing SB2 within the triple system TY CrA (Casey et al. 1998); a $1.1 M_{\odot}$ PMS star in the detached eclipsing SB2 EK Cep (Popper 1987); the eclipsing SB2 RS Cha, with masses of $1.86 M_{\odot}$ and $1.82 M_{\odot}$ (Anderson 1991), who was recently classified as PMS object by strong X-ray emission (Mamajek et al. 2000); and astrometric measurements of the PMS spectroscopic binary NTT 045251+3016 yielded masses of $1.4 M_{\odot}$ and $0.8 M_{\odot}$ (Steffen et al. 2000).

Due to the lack of a large sample of well determined stellar properties for stars in the PMS phase, the large discrepancies between the different sets of current PMS evolutionary models are not understood and the tracks could not be calibrated yet.

In order to explore if RX J1608.6-3922 is a spectroscopic binary and to confirm the eclipses, we carried out 
high-resolution spectroscopy and photometric monitoring and report here on the results of these observations.

\section{Data acquisition}

\subsection{Spectroscopy}

Using the Echelle spectrograph FEROS (Fiber fed Extended Range Spectrograph) on the $1.5 \mathrm{~m}$ telescope at La Silla, we have obtained 10 spectra of RX J1608.63922 in March and June 1999 and March 2000. FEROS is optimised for high precision measurements of radial velocities. The spectra cover the wavelength region between about $3600 \AA$ and $9200 \AA$ and have a resolution of $\lambda / \Delta \lambda=48000$. Precise radial velocities have been measured by cross-correlating the spectra of RX J1608.6-3922 with spectra of the radial velocity standard HR 5777, using the spectral range between 4000 and $6720 \AA$. The spectra of HR 5777 have been obtained also with FEROS in the same observing run. The radial velocity of this standard is known to be stable and has been determined with an accuracy of $55 \mathrm{~ms}^{-1}$ (Murdoch 1993).

\subsection{Photometry}

We monitored RX J1608.6-3922 photometrically in seven consecutive nights in April 2000 in Chile and Australia in the Johnson $V$ and $B$ filter. CCD images were obtained in Chile at the Danish $1.5 \mathrm{~m}$ telescope at ESO, La Silla, between April 21 and 28 with the imaging camera DFOSC. The observations in Australia were carried out with a CCD camera at the MSSSO 40 inch telescope at Siding Springs observatory between April 23 and 26. At MSSSO only $V$ band images have been taken.

We performed aperture photometry for RX J1608.63922 and several reference stars in the field with the qphot package of IRAF ${ }^{1}$. Differential photometry has been carried out, allowing us to compensate for variable atmospheric conditions and also to combine the Australian and Chilean data.

\section{Results}

\subsection{Radial velocities}

The main result of our spectroscopic observations is that we detected almost no variations of the radial velocity at all (see Fig. 1). The scatter does not exceed $2.4 \mathrm{~km} \mathrm{~s}^{-1}$ for 10 spectra obtained within one year. In particular this is the case for radial velocities measured in 5 consecutive nights (right panel, Fig. 1) covering more than half of the claimed period of 7.2 days. Hence, the semiamplitude of the detected variations cannot be larger than $2.4 \mathrm{~km} \mathrm{~s}^{-1}$ for a $7.2 \mathrm{~d}$ orbit.

\footnotetext{
1 IRAF is distributed by the National Optical Astronomy Observatories, which is operated by the Association of Universities for Research in Astronomy, Inc. (AURA) under cooperative agreement with the National Science Foundation.
}

This result comes as a surprise for a system showing (apparently) a primary and secondary eclipse of $0.5 \mathrm{mag}$ and $0.3 \mathrm{mag}$, respectively (W98). On the one hand only a very low-mass companion causes such small radial velocity variations, whereas on the other hand only a relatively luminous companion causes primary and secondary eclipses of almost similar amplitudes. Therefore it is unlikely that RX J1608.6-3922 is an eclipsing spectroscopic binary.

However, the detected radial velocity variations are significant but can easily be explained by stellar activity.

Based on the FEROS spectra, we determined the projected rotational velocity. Using the telluric lines for determining the instrumental profile of the spectrograph and assuming a solar-like center-to-limb variation, we derived a v sini of $22.4 \pm 1.9 \mathrm{~km} \mathrm{~s}^{-1}$. This is in agreement with the values of $21.8 \mathrm{~km} \mathrm{~s}^{-1}$ and $20 \mathrm{~km} \mathrm{~s}^{-1}$, estimated by Wichmann et al. (1999) by cross correlation and Fast Fourier Transformation, resp., using a template spectrum. Furthermore the radial velocity determined by these authors of $1.2 \mathrm{~km} \mathrm{~s}^{-1}$ agrees well with our values (cp. Fig. 1).

\subsection{The primary mass and the mass limit for a companion}

From the radial velocity variations an upper mass limit for an eclipsing companion can be derived. Assuming a mass of $M_{1}=0.78 M_{\odot}$ for the primary (Wichmann et al. 1997, hereafter W97) and an orbital period of $7.2 \mathrm{~d}$, a radial velocity semiamplitude of $2.4 \mathrm{~km} \mathrm{~s}^{-1}$ defines an upper mass limit of $20 M_{\text {Jup }}$ for any eclipsing companion to this star. The adopted primary mass was estimated by W97 from the star's bolometric luminosity and effective temperature and comparison with evolutionary tracks of D'Antona \& Mazzitelli (1994) using Canuto and Mazzitelli convection and Alexander opacities.

If there is a companion and it contributes significantly to the total luminosity of the system, the luminosity of the primary $L_{1}$ would be considerably smaller than the estimated luminosity of the unresolved binary. Considering the worst case, i.e. a secondary which is as luminous as the primary $\left(L_{1}=L_{2}=L_{\text {est }} / 2\right)$ and assuming similar spectral types, a comparison with the same evolutionary tracks, as used by W97, yields $M_{1} \approx 0.85 M_{\odot}$ and a correspondingly higher mass limit for a companion.

However, masses derived by means of comparison with current PMS evolutionary tracks are highly uncertain due to large discrepancies between different sets of theoretical PMS models and due to a lack of observational constraints. But even with a given evolutionary model, the error in the effective temperature of $\sim 200 \mathrm{~K}$ hamper an accurate placement into the Hertzsprung-Russell-diagram (HRD) and therefore an accurate mass determination. Taking the error in $T_{\text {eff }}$ into account gives a primary mass in the range of 0.6 to $1 M_{\odot}$ in the case of a neglectable secondary luminosity and 0.7 to $0.9 M_{\odot}$ if the secondary is as luminous as the primary $\left(L_{1}=L_{2}=L_{\text {est }} / 2\right)$. The error in the derived mass is smaller in the latter case because a 


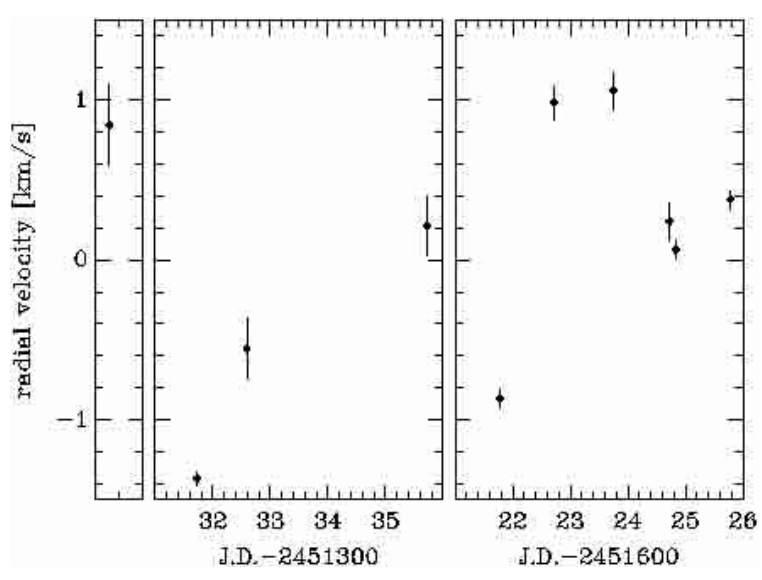

Fig. 1. Radial velocities of RX J1608.6-3922 determined from high-resolution spectra taken with FEROS between March 1999 and March 2000. The scatter of the radial velocity is smaller than $2.4 \mathrm{~km} \mathrm{~s}^{-1}$. The observations in 5 consecutive nights (right panel) cover more than half of the claimed period of $7.2 \mathrm{~d}$. Therefore these radial velocity measurements exclude a $7.2 \mathrm{~d}$ orbit with a higher radial velocity amplitude than $2.4 \mathrm{~km} \mathrm{~s}^{-1}$. The unlabeled data point in the left panel corresponds to a JD of 2451264.3 .

smaller luminosity shifts the position of the object in the HRD down to the radiative tracks, where the dependence on $T_{\text {eff }}$ is small.

To summarize, this means the primary mass $M_{1}$ can have values in the range of 0.6 to $1 M_{\odot}$, considering binarity and errors in $T_{\text {eff }}$, but not the errors in the adopted evolutionary tracks themselves. An eclipsing companion pulling on a $0.6 M_{\odot}$ primary star and causing its radial velocity to change as detected cannot be more massive than $17 M_{\text {Jup }}$. An eclipsing companion to a $1 M_{\odot}$ primary star causing the detected radial velocity scatter can have $24 M_{\text {Jup }}$ at the most. The absolute uncertainties in the mass determination of the primary star due to the use of different PMS evolutionary tracks should not be larger than a factor of two so that the possibly occulting object would still be substellar. In any case our spectroscopic observations allow only a very low-mass substellar companion.

\subsection{Light curves and color variations of RX J1608.6-3922}

We detected significant brightness variations of about $0.15 \mathrm{mag}$ in the $V$ band and $0.2 \mathrm{mag}$ in the $B$ band (see Fig. 2, top and middle). The obtained $V$ light curve does not reproduce the previously taken $V$ light curve by W98, specially we did not detect the deep minima showing a decrease of brightness of 0.3 and 0.5 mag. Therefore it is dubious whether the brightness variations recorded in 1996 by W98 can be explained by eclipses of a binary.

Periodic light variations observed from many T Tauri stars are interpreted as modulation of the total flux by spots on the stellar surface (see for example Bouvier et al. 1993, COYOTES I). The measured photometrical period
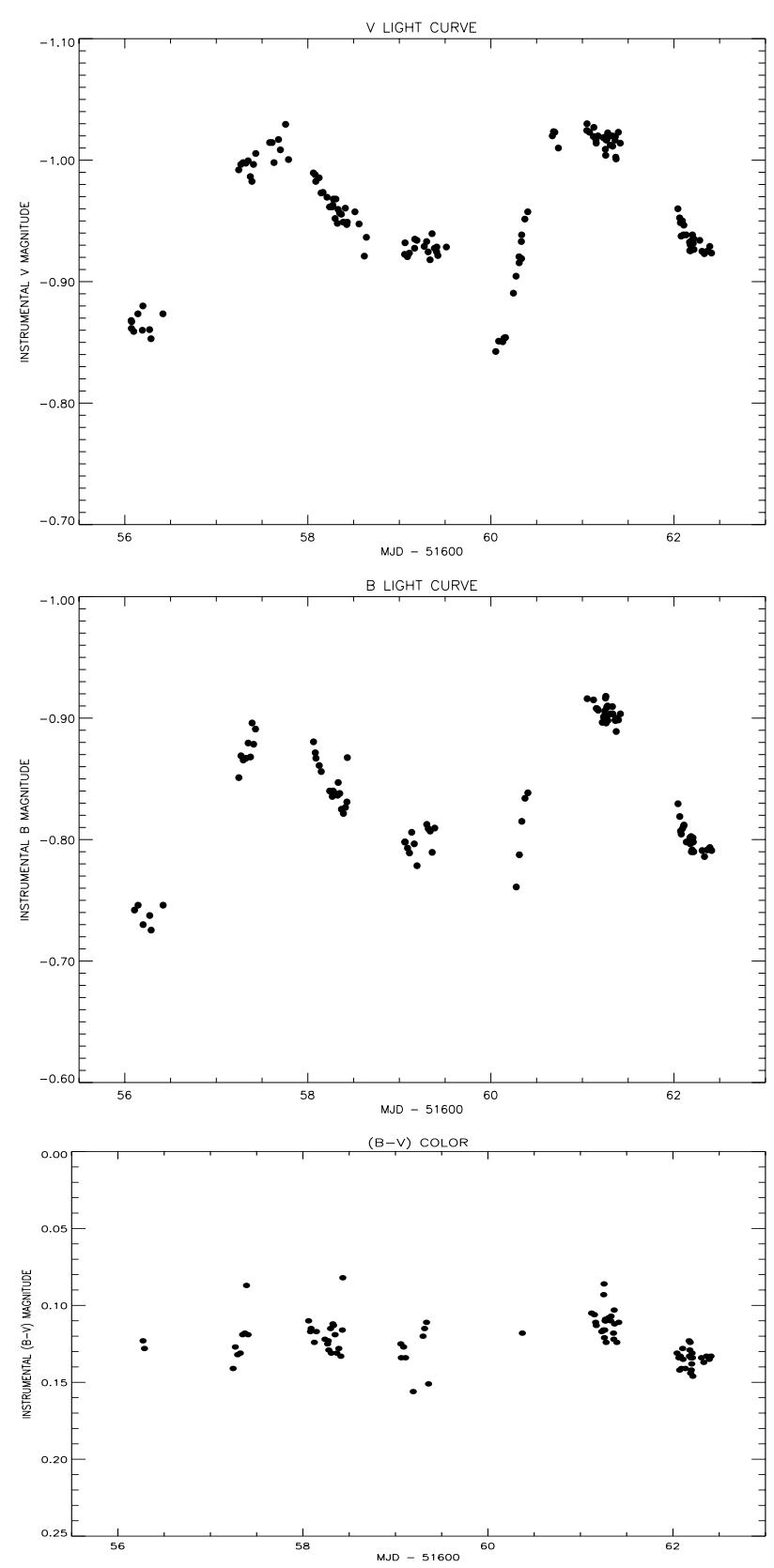

Fig. 2. Instrumental $V$ and $B$ magnitudes (top and middle) and instrumental $(B-V)$ colors (bottom) of RX J1608.6-3922 obtained at La Silla and Siding Springs. The brightness is increasing bottom-up. Both light curves show significant brightness variations. The amplitudes of the variations are higher at smaller wavelength (i.e. in the $B$ band). At Siding Springs only $V$ band measurements have been done, leading to larger gaps in the $B$ band light curve due to day time observing breaks. The $(B-V)$ values show small but significant color variations, indicating that the system is redder when darker.

is a direct tracer of the rotational period of the star. Such spot-driven photometric variations come along with color changes because of the different temperature and therefore spectral distribution of the spot regions compared to the surrounding photosphere.

For RX J1608.6-3922 we measure larger amplitudes of the brightness variations in the $B$ band than in the 
$V$ band, i.e. the emission of the system is shifted towards longer wavelength (= becoming redder) when the brightness decreases.

This observed behavior of the color is characteristic for cool as well as for hot spots. It can even be produced by the primary eclipse of a binary containing a relatively very low-mass companion, whereas the secondary eclipse of such a system will cause the opposite effect, that is becoming bluer when darker. A very clear reddening during primary eclipse and blueing during secondary eclipse was observed for TZ Mensae, a binary whose components have masses of $2.5 M_{\odot}$ and $1.5 M_{\odot}$ (Andersen et al. 1987).

The brightness of a single star with a cool spot on its surface is dimmed when the cool spot becomes visible and since it is cool and emitting more light at longer wavelength than at shorter the dimming is accompanied by reddening of the system.

A star with a hot spot shows minimum light when the hot spot is not visible and since the hot spot contributes more in the blue part of the spectral range, its disappearance is accompanied by relative reddening of the star-spot-system.

Eclipses in a system with a cool, low-mass companion to a hotter, more massive star can cause color variations along the cycle. During the primary eclipse, when the lowmass companion obscures (part of) the primary, the system gets darker and redder since (part of) the emission at shorter wavelength from the primary is missing.

During secondary eclipse, when the primary obscures the redder, low-mass companion, the system gets darker mainly in the red part of the spectral range and is therefore bluer in total during this phase.

In the lower panel of Fig. $2(B-V)$ values of RX J1608.6-3922 are shown. The $(B-V)$ color decreases when the star gets darker in $V$ and $B$, i.e. the star is redder when darker. This could be evidence for (cool or hot) spots or primary eclipses of a binary with a red, low-mass secondary. Based on the detected color variations we can exclude that the variations are due to a secondary eclipse.

We checked the photometric measurements taken in 1996 in the $V$ filter (W98) and the $B$ filter (Wichmann, pers. comm.) on color variations. Both minima show larger amplitudes in the $B$ band than in the $V$ band, therefore none of them can be explained by the secondary eclipse of a low-mass companion. However, a companion with a higher mass than $24 M_{\text {Jup }}$ is excluded by our spectroscopy (cp. Sect. 3.1).

\subsection{Period}

We used the string-length method (Dworetsky 1983) in order to search for periodicity in our data. Phase folding the data with a trial period and minimizing the stringlength between successive data points yields a minimum for the true period. Figure 3 displays string-length values for periods between 1 and 6 days. A clear minimum is

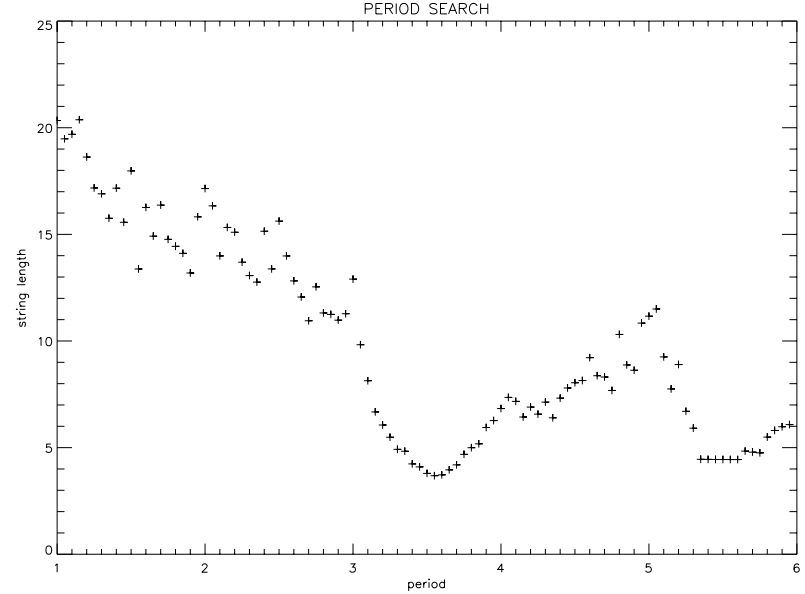

Fig. 3. Result of a search for periodicity in the $V$ photometry using the string-length method (Dworetsky 1983). Plotted are string-length values calculated for different periods in the interval from 1 to 6 days. Clearly visible is the minimum at 3.55 days. A second minimum is present at about $5.4 \mathrm{~d}$ but further analyses show that it is an alias.

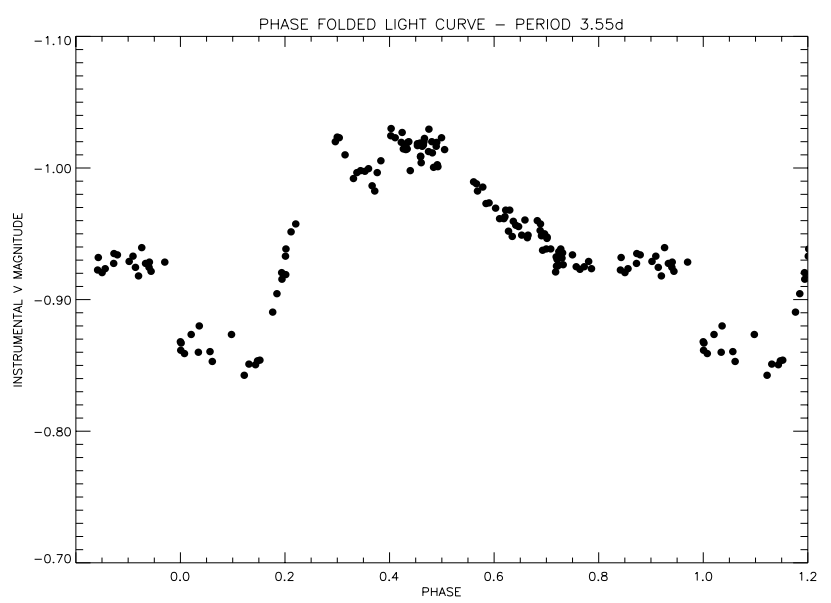

Fig. 4. Phase folded $V$ light curve of RX J1608.6-3922 for a period of 3.55 days. For clarity $20 \%$ more than one orbit has been plotted at the beginning as well as at the end of the light curve.

visible at $3.55 \mathrm{~d}$ and a second, less significant minimum at about $5.4 \mathrm{~d}$.

The light curve of RX J1608.6-3922 folded in phase with a $3.55 \mathrm{~d}$ period is displayed in Fig. 4 . It looks quite smooth. Phase folding the data with the less significant period of $5.4 \mathrm{~d}$ (cp. Fig. 5, bottom) makes clear that it is an alias.

Our photometric observations cover almost 7 days and we cannot search for periods longer than the total time basis, in particular not for the claimed period of $7.2 \mathrm{~d}$. However, phasing our $V$ magnitudes with $7.2 \mathrm{~d}$ shows that our data are also consistent with this period.

We phase folded the data taken by W98 with a period of $3.55 \mathrm{~d}$ and $7.2 \mathrm{~d}$ in order to compare them with our results. The folded W98 data are displayed in Fig. 6 . Although the $7.2 \mathrm{~d}$ period looks more convincing, a $3.55 \mathrm{~d}$ period is still in agreement with the data set. 

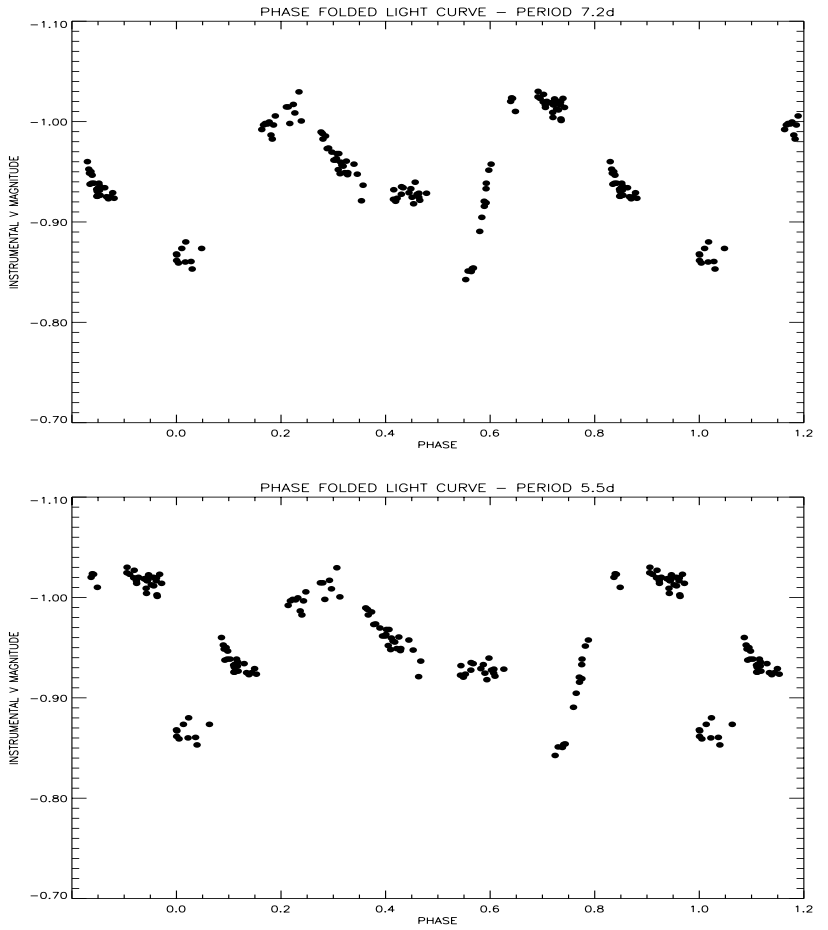

Fig. 5. Phase folded $V$ light curves of RX J1608.6-3922. In the upper panel the light curve is folded with a period of 7.2 days. This is the period claimed by W98. In the lower panel the light curve is folded with a period of 5.4 days. It clearly displays that the second, less significant period, found by means of the string-length method is an alias.

This means the photometric data of RX J1608.6-3922 from 1996 as well as from 2000 show brightness variations consistent with a $3.55 \mathrm{~d}$ as well as a $7.2 \mathrm{~d}$ period. If these flux modulations are caused by stellar spots the star is rotating with period of $3.55 \mathrm{~d}$ or $7.2 \mathrm{~d}$.

\subsection{Constraining the rotational period by means of the stellar radius and $v \sin i$}

An estimate of the rotational period for RX J1608.6-3922 based on the radius and the rotational velocity can help to distinguish between the two found periods. Stellar properties for RX J1608.6-3922 have been published by W97: They determined an effective temperature of $T_{\text {eff }}=4255 \mathrm{~K}$ and a luminosity of $\log \left(L_{*} / L_{\odot}\right)=-0.25$ for a distance of $140 \mathrm{pc}$. From these properties a radius of $R_{*}=1.38 R_{\odot}$ was derived. By comparison with evolutionary tracks from D'Antona \& Mazzitelli (1994) a mass of $M_{*}=0.78 M_{\odot}$ was estimated.

Based on our FEROS spectra, we determined the projected rotational velocity and found a $v \sin i$ of $22.4 \pm 1.9 \mathrm{~km} \mathrm{~s}^{-1}$. With unknown inclination $i$ of the star (we do not assume that the system is eclipsing) this value is a lower limit of the rotational velocity.

Based on $v \sin i$ and the radius of the star an upper limit of the rotational period can be derived:

$$
P_{\max }[\mathrm{d}]=50.6145 \frac{R\left[R_{\odot}\right]}{(v \sin i)\left[\mathrm{km} \mathrm{s}^{-1}\right]} .
$$
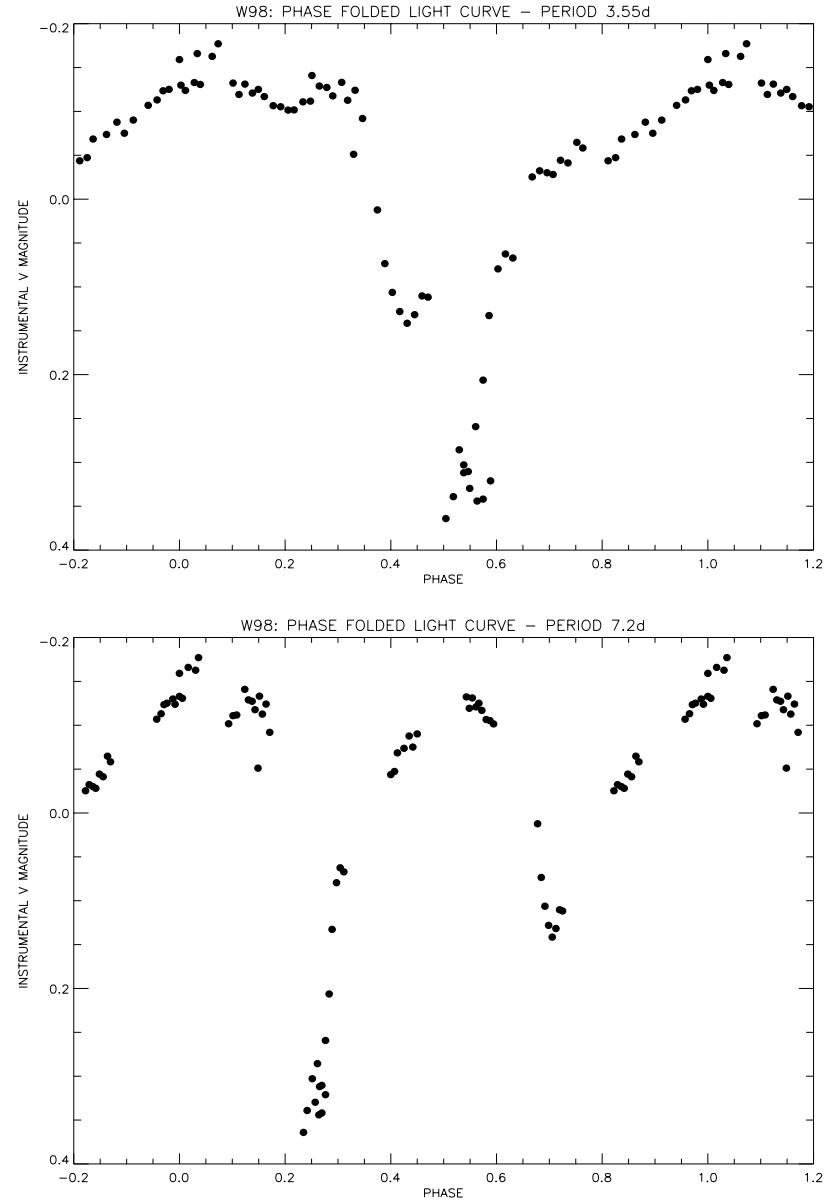

Fig. 6. Displayed are the photometric $V$ band data taken by W98 phase folded with a period of $3.55 \mathrm{~d}$ (top) and $7.2 \mathrm{~d}$ (bottom).

Adopting the radius of $1.38 R_{\odot}$ estimated by W97 the maximum period in the limiting case of an inclination of $90^{\circ}$ is $3.65 \mathrm{~d}$. This excludes definitely a rotational period of $7.2 \mathrm{~d}$.

W97 estimated the luminosity and therefore the radius for a distance of $140 \pm 20 \mathrm{pc}$ (for Lupus 1-4, Hughes et al. 1993). The Lupus complex consists of five clouds and RX J1608.6-3922 is located in Lupus 3 (for the location of the five Lupus clouds compare Tachihara et al. 1996). There are two other estimations of the distance to the Lupus star forming region based on HIPPARCOS measurements: Wichmann et al. (1998b) derive a considerably larger distance of $190 \pm 27$ pc, whereas Knude \& $\mathrm{H} \varnothing \mathrm{g}$ (1998) propose that at least Lupus 2-5 is as close as $100 \mathrm{pc}$.

The luminosity and therefore the radius depends crucially on the adopted distance. A larger distance gives a larger luminosity $L_{*}$ and therefore a larger radius $R_{*}$. Keeping the rotational velocity constant a larger radius stands for a longer period. Therefore in order to exclude $7.2 \mathrm{~d}$ as possible rotational period for RX J1608.6-3922 unambiguously, we must take into account the largest possible distance. 
Table 1. Stellar properties of RX J1608.6-3922. They are published by W97, except the following: spectral type (SpT) from Krautter et al. (1997), $v \sin i$ this paper, distances of the Lupus star forming region: $d_{\mathrm{K} \& \mathrm{H}}$ by Knude $\&$ Høg $(1998), d_{\text {Hughes93 }}$ by Hughes et al. (1993), $d_{\text {W98b }}$ by Wichmann et al. (1998b).

\begin{tabular}{|l|c|c|}
\hline property & value & error \\
\hline SpT & K6 & $0.8(1.6)$ subclasses \\
$V$ & $13.48 \mathrm{mag}$ & $0.01 \mathrm{mag}$ \\
$B-V$ & $1.52 \mathrm{mag}$ & $0.02 \mathrm{mag}$ \\
$U-B$ & $1.01 \mathrm{mag}$ & $0.02 \mathrm{mag}$ \\
$V-R_{\mathrm{C}}$ & $1.01 \mathrm{mag}$ & $0.02 \mathrm{mag}$ \\
$V-I_{\mathrm{C}}$ & $1.02 \mathrm{mag}$ & $0.02 \mathrm{mag}$ \\
$T_{\text {eff }}$ & $4255.0 \mathrm{~K}$ & $\Delta \log \left(T_{\text {eff }}\right)=0.02$ \\
$\log \left(L_{*} / L_{\odot}\right)$ & -0.25 & $\Delta \log \left(L_{*} / L_{\odot}\right) \approx 0.1$ \\
$A_{V}$ & $1.75 \mathrm{mag}$ & - \\
$R_{*} / R_{\odot}$ & 1.38 & $\Delta \log \left(R_{*} / R_{\odot}\right) \approx 0.2$ \\
$M_{*} / M_{\odot}$ & 0.78 & - \\
$\log ($ age $)$ & $6.47 \mathrm{yr}$ & - \\
$(v \sin i)$ & $22.4 \mathrm{~km} \mathrm{~s}$ & $1.9 \mathrm{~km} \mathrm{~s}{ }^{-1}$ \\
$d_{\mathrm{Hughes} 93}$ & $140 \mathrm{pc}$ & $20 \mathrm{pc}$ \\
$d_{\mathrm{W} 98 \mathrm{~b}}$ & $190 \mathrm{pc}$ & $27 \mathrm{pc}$ \\
$d_{\mathrm{K} \& \mathrm{H}}$ & $100 \mathrm{pc}$ & - \\
\hline
\end{tabular}

\subsubsection{Radius estimate from $L_{*}$ and $T_{\text {eff }}$}

The radius of a star can be calculated from $T_{\text {eff }}$ and $L_{*}$

$L_{*}=4 \pi R_{*}^{2} \sigma T_{\mathrm{eff}}^{4}$

and depends on the apparent bolometric magnitude $m_{\text {bol }}$, the distance $d$ and the extinction $A_{V}$.

$\log \left(L_{*} / L_{\odot}\right)=\frac{\left(4.74-m_{V}+A_{V}-B C+5 \log d-5\right)}{2.5}$

$\log \left(L_{*} / L_{\odot}\right)=-4.468+2 \log d[\mathrm{pc}]$.

We adopt a bolometric correction of $B C=-0.82 \pm 0.1$, as given by Kenyon \& Hartmann (1995) for a star with spectral type K6. The error of $B C$ is about 0.1 due to the given (W97) error of $\sim 200 \mathrm{~K}$ in $T_{\text {eff }}$. The error in $A_{V}$ is about $0.3 \mathrm{mag}$ due to errors in the intrinsic color as well as the applied conversion table. The luminosity is estimated for distances of $100 \pm 20 \mathrm{pc}, 140 \pm 20 \mathrm{pc}$ and $190 \pm 27 \mathrm{pc}$, respectively:

$\log \left(L_{*} / L_{\odot}\right)_{100}=-0.47 \pm 0.34$

$\log \left(L_{*} / L_{\odot}\right)_{140}=-0.18 \pm 0.29$

$\log \left(L_{*} / L_{\odot}\right)_{190}=0.09 \pm 0.29$.

Applying the Stefan-Boltzmann law (2) yields the radius:

$$
\begin{array}{r}
R_{*}\left[R_{\odot}\right]=\frac{1}{R_{\odot}[\mathrm{m}]} \cdot \sqrt{\frac{L_{*}\left[L_{\odot}\right] \cdot L_{\odot}[W]}{4 \pi \sigma\left[\frac{\mathrm{W}}{\mathrm{m}^{2} \mathrm{~K}^{4}}\right]\left(T_{\mathrm{eff}}^{4}\left[\mathrm{~K}^{4}\right]\right)}} \\
R_{*}\left[R_{\odot}\right]=3.3379 \times 10^{7} \cdot \sqrt{\frac{L_{*}\left[L_{\odot}\right]}{T_{\mathrm{eff}}^{4}\left[\mathrm{~K}^{4}\right]}}
\end{array}
$$

$R_{*, 100}=(1.07 \pm 0.33) R_{\odot} \quad$ for $100 \pm 20 \mathrm{pc}$

$R_{*, 140}=(1.50 \pm 0.36) R_{\odot} \quad$ for $140 \pm 20 \mathrm{pc}$

$R_{*, 190}=(2.04 \pm 0.49) R_{\odot} \quad$ for $190 \pm 27 \mathrm{pc}$.
The maximum period for these radii in the limiting case of an inclination of $90^{\circ}$ would be

$$
\begin{aligned}
& P_{\max , 100}=(2.4 \pm 0.9) \mathrm{d} \\
& P_{\max , 140}=(3.4 \pm 1.1) \mathrm{d}
\end{aligned}
$$$$
P_{\max , 190}=(4.6 \pm 1.5) \mathrm{d} \text {. }
$$

Therefore the largest possible radius would be $2.53 R_{\odot}$ (for a distance of $(190+27) \mathrm{pc}$ ) and the largest possible upper limit for the rotational period would be $P_{\max }=6.1 \mathrm{~d}$, excluding a $7.2 \mathrm{~d}$ period.

\subsubsection{Radius estimate from Barnes-Evans type relation for $\mathrm{K}$ giants}

Beuermann et al. (1999, hereafter B99) published recently Barnes-Evans type relations for late-type giants and dwarfs. Their results for giants are based on angular diameters measured for $27 \mathrm{M}$-giants and $9 \mathrm{~K}$-giants. The dwarf relation is based on the measurement of the angular diameter for YY Gem (eclipsing binary) and of estimations of angular diameters for $8 \mathrm{M}$-dwarfs. The latter are derived by flux fitting of model atmospheres to observed low-resolution optical/IR spectra (for details see B99 and references therein).

The B99 relations are based on $V-I_{\mathrm{C}}$ measurements and allow us to directly use the $V-I_{\mathrm{C}}$ color of $1.02 \mathrm{mag}$ measured for RX J1608.6-3922 (W97). The star's luminosity class is $\mathrm{V}$ or IV (Wichmann, pers. comm.), i.e. being a dwarf or an intermediate object between a dwarf and a giant.

The radius can be estimated directly from the absolute magnitude $M_{\lambda}$ and the visual surface brightness $S_{\lambda}$ :

$S_{\lambda}=M_{\lambda}+5 \log \left(R_{*} / R_{\odot}\right)$

B99 derived linear relationships between $S_{\lambda}$ and $V-I_{\mathrm{C}}$ for M-giants as well as for M-dwarfs. A given relationship for K-giants is based on a linear fit to $9 \mathrm{~K}$-giants $\left(V-I_{\mathrm{C}}<1.65\right)$ tied to the Sun:

$S_{V, \text { Kgiants }}=2.86+2.84\left(V-I_{\mathrm{C}}\right)$ for $V-I_{\mathrm{C}}<1.65$.

The argument for including the Sun is that Barnes \& Evans (1976) and Barnes et al. (1977) found that there is no difference in the surface brightness of giants and dwarfs for stars of spectral type B-G and that the Sun falls on the giant relation.

The absolute magnitude $M_{V}$ for RX J1608.6-3922 is:

$$
\begin{array}{r}
\Delta M_{V}=0.31+\frac{5 \Delta d}{d \ln 10} \\
M_{V}=6.73 \pm 0.74 \text { for } d=100 \mathrm{pc} \\
M_{V}=6.00 \pm 0.62 \text { for } d=140 \mathrm{pc} \\
M_{V}=5.34 \pm 0.62 \text { for } d=190 \mathrm{pc} .
\end{array}
$$

B99 provides no relation for dwarfs with $\left(V-I_{\mathrm{C}}\right)<1.65$, as is the case for RX J1608.6-3922. The giant-relation Eq. (7) may nevertheless be used since an object with $\left(V-I_{\mathrm{C}}\right)=$ 1.02 should be well in the regime where the authors expect 
the separation between both relations to be very small. The radius estimated with Eqs. (6) and (7) is:

$$
\begin{array}{cc}
R_{*, \mathrm{Kgiants}}=(0.64 \pm 0.32) R_{\odot} & \text { for } 100 \pm 20 \mathrm{pc} \\
R_{*, \mathrm{Kgiants}}=(0.89 \pm 0.40) R_{\odot} & \text { for } 140 \pm 20 \mathrm{pc} \\
R_{*, \mathrm{Kgiants}}=(1.21 \pm 0.54) R_{\odot} & \text { for } 190 \pm 27 \mathrm{pc} .
\end{array}
$$

The errors have been estimated by adopting an error of $6 \%$ in $S_{V}$ (cp. B99). The maximum rotational periods in the limiting case of an inclination of $90^{\circ}$ for these radii are:

$$
\begin{gathered}
P_{\max , 100}=1.4 \mathrm{~d} \pm 0.8 \mathrm{~d} \\
P_{\max , 140}=2.0 \mathrm{~d} \pm 1.1 \mathrm{~d} \\
P_{\max , 190}=2.7 \mathrm{~d} \pm 1.4 \mathrm{~d} .
\end{gathered}
$$

The radii estimated with the B99-K-giant-relation are smaller than the radii derived from the luminosity and the effective temperature and consequently also the rotational periods. The largest possible upper limit for the rotational period with these radius estimates is $P_{\max }=4.1 \mathrm{~d}$, also excluding a $7.2 \mathrm{~d}$ period and favoring the $3.55 \mathrm{~d}$ period.

\section{Discussion and conclusions}

It was claimed by W98 that the T Tauri star RX J1608.63922 is an eclipsing binary. We reported here on results of high-resolution spectroscopy and photometric monitoring of this object.

The measured radial velocities of RX J1608.6-3922 are almost constant. Our measurements cover suitable time spans in order to detect a binary with a 7 day period at the maximum separation phases. The scatter of the radial velocities does not exceed $2.4 \mathrm{~km} \mathrm{~s}^{-1}$, giving evidence that it is not an eclipsing binary as claimed previously by W98. An eclipsing companion to a 0.6 to $1 M_{\odot}$ star, orbiting the central object with a $7.2 \mathrm{~d}$ period and causing the measured radial velocity variations with an amplitude of $2.4 \mathrm{~km} \mathrm{~s}^{-1}$, would be less massive than $24 M_{\mathrm{Jup}}$.

$V$ and $B$ band photometry obtained in 7 consecutive nights in Chile and Australia shows brightness variations of the order of $0.15 \mathrm{mag}$ in $V$ and $0.2 \mathrm{mag}$ in $B$. The shape of these light curves obtained by us in 2000 differs significantly from the shape of the light curves obtained by W98 in 1996. In particular the deep minima of $0.5 \mathrm{mag}$ and 0.3 mag observed in 1996 are not detected by us. Therefore the brightness variations recorded in 1996 cannot be explained by eclipses of a binary system.

Another argument against the eclipsing binary hypothesis is the behaviour of the color variations, since the object is redder during minimum light. This behaviour is characteristic for (cool and hot) spots, but can also be caused by the primary eclipse of a binary with a very red, low-mass companion. The secondary eclipse of such a system would show the opposite color variation, i.e. becoming bluer during minimum light. Such a behaviour is not observed during the 7 nights.

We also checked the $V$ and $B$ band data recorded in 1996 (W98, $B$ data: pers. comm.) on color variations. The emission of the object is shifted during both minima towards longer wavelength and therefore none of the minima is explicable by a secondary eclipse of a low-mass companion. However, a companion with similar mass as the primary would not show color variations at all but this is in any case definitely excluded by our spectroscopic observations.

Concerning the rotational period of the star we found that our photometric data are periodic with about $3.6 \mathrm{~d}$. These data are also consistent with the period of $7.2 \mathrm{~d}$ found by W98. On the other hand the W98 data are also in agreement with a $3.6 \mathrm{~d}$ period, apart from small deviations.

We estimated an upper limit for the rotational period based on the radius and the projected rotational velocity. We took into account different distance estimates for the Lupus star forming region, as well as cross checked the radius estimate with different methods. In any case the rotational period of RX J1608.6-3922 does not exceed a duration of 6.1 days, favoring $3.6 \mathrm{~d}$ as the true rotational period.

The radius was estimated with two different methods: once from the luminosity and the effective temperature and once by means of Barnes-Evans-type relations published by B99. The results agree within the measurements uncertainties but the B99-radii are systematically smaller. Depending on the adopted distance the radius is $1.07 R_{\odot}\left(1.50 R_{\odot}, 2.04 R_{\odot}\right)$ for $d=100 \mathrm{pc}(140 \mathrm{pc}, 190 \mathrm{pc})$ based on the $L_{\mathrm{bol}}-T_{\text {eff }}$ estimation and $0.64 R_{\odot}\left(0.89 R_{\odot}\right.$, $\left.1.21 R_{\odot}\right)$, respectively, based on the B99-estimation. Since $v \sin i$ for the star is $22.4 \mathrm{~km} \mathrm{~s}^{-1}$, a rotational period of $3.6 \mathrm{~d}$ allows the radius not to be smaller than $1.6 R_{\odot}$. These considerations suggest a radius for RX J1608.6-3922 in the range of 1.6 to $2.5 R_{\odot}$. It should be mentioned that this result is not compatible with a distance of $100 \mathrm{pc}$.

The variations of the photometric brightness of RX J1608.6-3922 are interpreted as modulation due to active, spotted regions on the stellar surface. They probably also account for the observed radial velocity scatter of $2.4 \mathrm{~km} \mathrm{~s}^{-1}$.

Krautter et al. (1997) classify RX J1608.6-3922 as a weak line $\mathrm{T}$ Tauri star on basis of an $\mathrm{H}_{\alpha}$ equivalent width of $7.4 \AA$ (spectrum obtained in the time span between 1991 and 1993). Wichmann et al. (1999) measured an equivalent width of $14.2 \AA$ in 1995 , based on high-resolution spectroscopy. These measurements suggest that the $\mathrm{H} \alpha$ emission of this star is variable and that it might well be a classical T Tauri star (cTTS). The accretion of circumstellar material onto the surface of a star can cause dominant hot spots. Provided that RX J1608.6-3922 is a cTTS, the detected variability can be caused by such hot spots and regardless of the presence of circumstellar material the photometric variability can be caused by cool spots.

The variable shape of the light curve indicates that the spotted regions change their properties (size, temperature and/or location) in time scales of a few years. 
Variable shapes of light curves are observed for many active stars, like RS CVn and BY Dra stars. For RS CVn stars it is known that their light curves change in shape, mean light level and amplitude on time scales of a year or even more rapid (e.g. V 711 Tau, Kang \& Wilson 1989, $\sigma$ Gem, Oláh et al. 1989, DM UMa, Mohin \& Raveendran 1992, HK Lac, Oláh et al. 1997). This is interpreted as rotational modulation due to cool spots on the surface, which rapidly change their location, size and/or temperature.

Analysis of the evolution of spots for several pre-mainsequence stars by Grankin (1998) (and references therein) showed that the initial epochs and the rotational periods are stable on time scales of several years, whereas the shape and amplitude of the light curves changes from year to year. The stability of the rotational periods are explained by stable positions of cool, spotted regions confined to a specific active longitudinal region. The variations of the spot light curve in shape and amplitude can then be explained by the appearance, disappearance and migration of individual spots. This scenario is also supported by the work of Schüssler \& Solanki (1992), who demonstrate that in rapidly rotating stars, the strong curiolis forces push the magnetic flux always towards the poles. Therefore the observations of cool spots remaining at the same position for several years on $\mathrm{T}$ Tauri stars can be explained by stable active regions in which new spots are produced continuously.

For V 410 Tau photometric observations over the time span 1981 to 1997 (Vrba et al. 1988; Herbst 1989; Grankin 1999) show that the photometric behaviour of this system is characterized by year-to-year variations of the shape and amplitude of the light curve. Modeling of the spot distribution based on photometry (Herbst 1989), as well as Doppler imaging of the surface structure (Hatzes 1995 and references therein) indicate that there is a stable highlatitude spot area lasting for at least several years.

Another example for a $\mathrm{T}$ Tauri star with indications for a long lasting stable spot distribution is P1724 (Neuhäuser et al. 1998 and references therein). A periodicity of $5.7 \mathrm{~d}$ is present in photometric observations covering almost 30 years and can even be traced in radial velocity measurements covering about 1.5 years. The relative phasing between the photometric and the spectroscopic data is almost stable over the entire time span of the spectroscopic observations, suggesting a basically non-varying spot distribution.

We conclude that RX J1608.6-3922 is a spotted star with a rotational period of about 3.6 days and that spots have mimicked the light-curve of an eclipsing binary in the data which were previously obtained. There are indications that the stellar activity on RX J1608.6-3922 is highly variable, with an equivalent width of the $\mathrm{H}_{\alpha}$ emission changing from about 7 to about $14 \AA$ within a few years and a variable shape of the light curve, with amplitudes changing from about 0.5 to about $0.2 \mathrm{mag}$ within 4 years. Therefore RX J1608.6-3922 is an interesting object for further study of stellar activity on $\mathrm{T}$ Tauri stars.
Acknowledgements. We acknowledge helpful discussions on the topic of this paper with G. Wuchterl, N. Huélamo and K. Fuhrmann. VJ acknowledges grant from the Deutsche Forschungsgemeinschaft (Schwerpunktprogramm "Physics of star formation"). RN acknowledges financial support from the Bundesministerium für Bildung und Forschung through the Deutsche Zentrum für Luft- und Raumfahrt e.V. (DLR).

\section{References}

Anderson, J., Clausen, J. V., \& Nordström, B. 1987, A\&A, 175,60

Anderson, J. 1991, A\&ARv, 3, 91

Barnes, T. G., \& Evans, D. S. 1976, MNRAS, 174, 489

Barnes, T. G., Dominy, J. F., Evans, D. S., et al. 1977, MNRAS, 178, 661

Beuermann, K., Baraffe, I., \& Hauschildt, P. 1999, A\&A, 348, 524 (B99)

Bouvier, J., Cabrit, S., Fernández, M., Martín, E. L., \& Matthews, J. M. 1993, A\&A, 272, 176

Casey, B. W., Mathieu, R. D., Vaz, L. P. R., Andersen, J., \& Suntzeff, N. B. 1998, AJ, 115, 1617

Covino, E., Catalano, S., Frasca, A., et al. 2000, A\&A, 361, L49

D'Antona, F., \& Mazzitelli, I. 1984, A\&A, 138, 431

Dworetsky, M. M. 1983, MNRAS, 203, 917

Grankin, K. N. 1998, Astron. Lett., 24(4), 497

Grankin, K. N. 1999, Astron. Lett., 25(8), 526

Hatzes, A. P. 1995, ApJ, 451, 784

Herbst, W. 1989, AJ, 98, 2268

Hughes, J., Hartigan, P., \& Clampitt, L. 1993, AJ, 104, 680

Kang, Y. W., \& Wilson, R. E. 1989, AJ, 97, 848

Kenyon, S. J., \& Hartmann, L. 1995, ApJS, 101, 117

Knude, J., \& Høg, E. 1998, A\&A, 338, 897

Krautter, J., Wichmann, R., Schmitt, J. H. M. M., et al. 1997, AAS, 123,329

Mamajek, E. E., Lawson, W. A., \& Feigelson, E. D. 2000, ApJ, 544,356

Mohin, S., \& Raveendran, A. V. 1992, A\&A, 256, 487

Murdoch, K. A., Hearnshaw, J. B., \& Clark, M. 1993, ApJ, 413,349

Neuhäuser, R., Wolk, S. J., Torres, G., et al. 1998, A\&A, 334, 873

Oláh, K., Panov, K. P., Pettersen, B. R., Valtaoja, E., \& Valtaoja, L. 1989, A\&A, 218, 192

Oláh, K., Kövári, Zs., Bartus, J., et al. 1997, A\&A, 321, 811

Popper, D. M. 1987, ApJ, 313, L81

Schüssler, M., \& Solanki, S. K. 1992, A\&A, 264, L13

Steffen, A. T., Mathieu, R. D., Lattanzi, M. G., et al. 2000, AAS, 196405

Tachihara, K., Dobashi, K., Mizuno, A., Ogawa, H., \& Fukui, Y. 1996, PASJ, 48, 489

Vrba, F. J., Herbst, W., \& Booth, J. F. 1988, AJ, 96, 1032

Wichmann, R., Krautter, J., Covino, E., et al. 1997, A\&A, 320, 185 (W97)

Wichmann, R., Bouvier, J., Allain, S., \& Krautter, J. 1998, A\&A, 330, 521 (W98)

Wichmann, R., Bastian, U., Krautter, J., Jankovics, I., \& Ruciǹski, S. M. 1998b, MNRAS, 301, L39

Wichmann, R., Covino, E., Alcalá, J. M., et al. 1999, MNRAS, 307, 909 